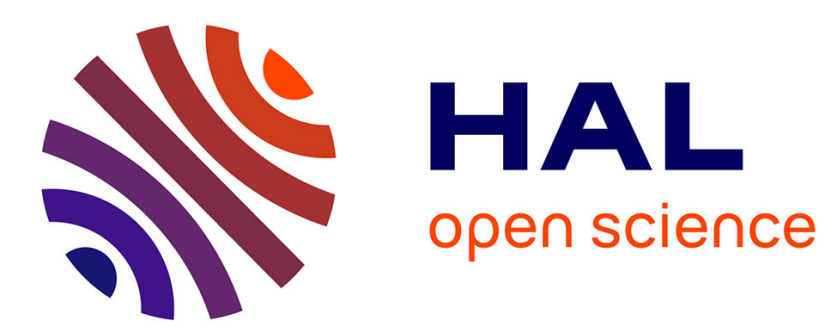

\title{
Four algorithms to construct a sparse kriging kernel for dimensionality reduction
}

Christophette Blanchet-Scalliet, Céline Helbert, Mélina Ribaud, Céline Vial

\section{To cite this version:}

Christophette Blanchet-Scalliet, Céline Helbert, Mélina Ribaud, Céline Vial. Four algorithms to construct a sparse kriging kernel for dimensionality reduction. Computational Statistics, 2019, 34, pp.1889-1909. 10.1007/s00180-019-00874-2 . hal-01496521v3

\section{HAL Id: hal-01496521 \\ https://hal.science/hal-01496521v3}

Submitted on 4 Sep 2018

HAL is a multi-disciplinary open access archive for the deposit and dissemination of scientific research documents, whether they are published or not. The documents may come from teaching and research institutions in France or abroad, or from public or private research centers.
L'archive ouverte pluridisciplinaire HAL, est destinée au dépôt et à la diffusion de documents scientifiques de niveau recherche, publiés ou non, émanant des établissements d'enseignement et de recherche français ou étrangers, des laboratoires publics ou privés. 


\title{
Four algorithms to construct a sparse kriging kernel for dimensionality reduction
}

\author{
Christophette Blanchet-Scalliet ${ }^{1}$, Céline Helbert ${ }^{1}$, Mélina Ribaud ${ }^{* 1}$, and Céline \\ $\mathrm{Vial}^{2,3}$ \\ ${ }^{1}$ Univ Lyon, École centrale de Lyon, CNRS UMR 5208, Institut Camille Jordan, \\ 36 avenue Guy de Collonge, F-69134 Ecully Cedex, France \\ ${ }^{2}$ Univ Lyon, Université Claude Bernard Lyon 1, CNRS UMR 5208, Institut \\ Camille Jordan, 43 blvd. du 11 novembre 1918, F-69622 Villeurbanne cedex, \\ France \\ ${ }^{3}$ INRIA, Villeurbanne, France
}

April 9, 2018

\begin{abstract}
In the context of computer experiments, metamodels are largely used to represent the output of computer codes. Among these models, Gaussian process regression (kriging) is very efficient see e.g Snelson (2008). In high dimension that is with a large number of input variables, but with few observations the estimation of the parameters with a classical anisotropic kriging becomes completely wrong. The number of the parameters to estimate is the same as the number of inputs and it implies that the space of the optimization becomes too big compare to the available informations. One way to overcome this drawback is to use the isotropic kernel which is more robust because it estimates not as many parameters. However this model is too restrictive. The aim of this paper is twofold. Our first objective is to propose a smooth kernel with as few parameters as warranted. We propose a kernel which is a tensor product of few isotropic kernels built on well-chosen subgroup of variables. The main difficulty is to find the number and the composition of groups. Our second objective is to propose algorithmic strategies to overcome the difficulty of finding the number and the composition of the groups. Four forward strategies are proposed. They all start with the simplest isotropic kernel and stop when the best model according to BIC criterion is found. They all show very good accuracy results on simulation test cases. But one of them is the most efficient. Tested on a real data set, our kernel shows very good prediction results.
\end{abstract}

Keywords. metamodel, isotropic, anisotropic, clustering

*melina.ribaud@doctorant.ec-lyon.fr 


\section{Introduction}

Review : Réorganiser le paragraphe en italique, je vois pas trop comment réorganiser.

Complex physical phenomena are more and more studied through numerical simulations. These numerical models are able to mimic with a high accuracy the real experiments so they predict the physical measures of interest (outputs) very precisely. Then, we can use them as a replacement for real experiments because the numerical simulations are less costly in primary materials. However, these simulations stay often time-consuming.

The idea to overcome this drawback is to replace the costly numerical model by a metamodel. A metamodel is a less expensive model adjusted on a few well-chosen simulations. It can be shown that among all the possible metamodels, Gaussian process regression (kriging) is a very efficient metamodel. Lots of examples of the use of a metamodel can be found in litterature, see e.g. Marrel et al. (2008), Antoniadis et al. (2012) and Sudret (2012). A detailed example of kriging is the helicopter test displayed in Booker et al. (1998).

Furthermore Villa-Vialaneix et al. (2012) shows a comparative study of eight metamodeling techniques for the simulation of $\mathrm{N}_{2} 0$ fluxes and $N$ (Nitrogen) leaching from corn crops. In this context, Splines and kriging have the best performances for small and medium training datasets. In addition, kriging is able to model highly complex data, see e.g. Santner et al. (2003) and Rasmussen and Williams (2006). More precisely kriging is a spatial interpolation technique which aims at predicting the outputs using an adapted underlying correlation function between design points. In fact we assume that the output of interest is a realization of a Gaussian process (GP) constructed as a sum of a deterministic part (often called the trend) and a stochastic part assumed to be a zero-mean stationary GP, see Roustant et al. (2012).

In this paper, we focus on the stochastic part and more precisely on the choice of the covariance kernel structure. In general, people use an anisotropic kernel that is a tensor product of as many $1 D$ kernels as the number of inputs. Each kernel being parameterized by a spatial correlation length, called the range parameter. In high dimension with a very restricted number of data points the estimation of range parameters becomes quite difficult. That's why in this context, an isotropic kernel could be a good alternative. This kernel is a function of the euclidean distance defined on the entire input space, so it depends only on one range parameter. However isotropic kernels are too restrictive, given that spatial variations are controlled by only one range parameter. The idea of this paper is to automatically construct a data-driven kernel that is intermediate between these two extremal choices.

A review of the literature shows that one way to improve performance of kriging is to adapt the covariance structure to each specific case, see e.g. Durrande (2001) and Ginsbourger et al. (2016). For example Paciorek and Schervish (2006) create a new class of covariance functions (kernels) allowing the model to adapt itself to spatial surface whose variability changes with location. Likewise, Padonou and Roustant (2016) in a microelectronic framework define a GP model which inserts the geometry of the wafer in the kernel. That's why they introduce the polar GP defined with respect to polar coordinates: the covariance function is a sum of a product kernel of radius and a product kernel of polar angles. In the case of multiple outputs Fricker et al. (2013) define a nonseparable covariance structure for GP with the aim of well representing the different simulator outputs and the joint uncertainty. In our case, the idea is to use a covariance structure adapted to high dimension 
In general the covariance function only depends on the range parameters and on the sill parameter (variance) which have to be estimated. But range parameters estimation becomes very tricky in high dimension. To overcome this difficulty, Yi (2009) proposed a penalized kriging that mimics the penalization techniques used in linear regression. In fact, he penalizes the likelihood by the norm of the range parameters vector. This methodology selects the variables that have no effect on the covariance function and the associate range parameters are blowing up to infinity. In order to solve the dimensionality of the problem, Binois (2015) proposes different sparse and specific covariance kernels based on expert information or on the work of Muehlenstaedt et al. (2012) and Durrande et al. (2012). In these articles, they use the ANOVA kernels that are a sum of sub-groups of variables that interact together, see more details in Stitson et al. (1997) and Gao et al. (2002). Muehlenstaedt et al. (2012) found these groups through a sensitivity analysis computed from the prediction function of an anisotropic kriging. A first problem appears here. Because the number of points is often too restrictive, the quality of the anisotropic kriging can be poor and the result of the sensitivity analysis such as the proposed partition can be totally wrong. A second problem of the method proposed by Muehlenstaedt and coauthors is that the number of parameters of the final kernel can be higher than that of the anisotropic kriging. In Binois et al. (2015) they test different FANOVA constraining them to have fewer range parameters than the anisotropic one and choosing the best kernel among them. But this choice of the covariance structure can not be done automatically.

Finally, we can also find in literature the work of Welch et al. (1992) that aims at identifying the active factors. Welch et al. (1992) proposes a likelihood-based forward algorithm to determine the most important factors and to build the predictor. In this study a tensor product of power exponential kernels is chosen to catch the function regularity. The dimension of the kernel, i.e. two parameters (range and power) in each direction, is stepwise reduced by making some range parameters equal. In our paper the structure of the kernel is different. It is a tensor product of isotropic kernels by taking an euclidean norm for the distance in each subspace. We propose to use a Matern kernel of order $5 / 2$ that needs only one parameter (range), that models intermediate regularity functions and that gives efficient and good results in industrial cases, see Cornford et al. (2002).

The aim of our article is to propose four algorithms that automatically construct from data a sparse kernel adapted to high dimension. The article is structured as follows. In section 2, we introduce the kriging metamodel and the different kernels. In section 3 , we present the four algorithms that automatically select the structure of the kernel according to the data. In section 4 , we study the behavior of our algorithms on simulation test cases.

\section{Statistical models}

This section introduce the kriging, anisotropic and isotropic kernels and finally the new general class of isotropic by group kernel.

\section{$2.1 \quad$ Kriging}

Let $p$ be the number of input variables. We consider $n$ observations $\left(\mathbf{x}_{i}, y_{i}\right)_{i=1, \ldots, n}$ where $\mathbf{x}_{i}$ is the ith input vector with $p$ coordinates, such that $\mathbf{x} \in \mathbb{R}^{p}$ and where $y_{i}$ is the corresponding output $\left(y_{i} \in \mathbb{R}\right)$. In the following we denote by $\mathbf{y}=\left(y_{1}, \ldots, y_{n}\right)^{\prime}$ the vector of the outputs. In kriging 
we assume that $\mathbf{y}$ is a realization of a Gaussian process $(Y(\mathbf{x}))_{\mathbf{x} \in \mathbb{R}^{p}}$ at points $\left(\mathbf{x}_{1}, \ldots, \mathbf{x}_{n}\right)^{\prime}$ such that for each $x \in \mathbb{R}^{p}$ :

$$
Y(\mathbf{x})=m+\epsilon(\mathbf{x})
$$

where $m \in \mathbb{R}$ is the trend, the process $(\epsilon(\mathbf{x}))_{\mathbf{x} \in \mathbb{R}^{p}}$ is a centered stationary Gaussian process with covariance function $\operatorname{Cov}\left(\epsilon(\mathbf{x}), \epsilon\left(\mathbf{x}^{\prime}\right)\right)=\sigma^{2} R\left(\mathbf{x}, \mathbf{x}^{\prime}\right)=\sigma^{2} \mathbf{r}\left(\mathbf{x}-\mathbf{x}^{\prime}\right), \forall\left(\mathbf{x}, \mathbf{x}^{\prime}\right) \in \mathbb{R}^{p} \times \mathbb{R}^{p}$. In this paper the trend $m$ and the variance $\sigma^{2}$ are assumed to be constant.

In this context we want to construct a linear prediction that minimizes the mean-squared prediction error and that guarantees uniform unbiasedness. Under these two constrains, the prediction, see Cressie (1993)), at a point $\mathbf{x}_{\mathbf{0}} \in \mathbb{R}^{p}$ is given by :

$$
\widehat{Y}\left(\mathbf{x}_{\mathbf{0}}\right)=m+\mathbf{r}\left(\mathbf{x}_{\mathbf{0}}\right)^{\prime} \boldsymbol{R}^{-1}\left(\mathbf{y}-m \mathbb{1}_{n}\right)
$$

and the Mean Square Error (MSE) at point $\mathbf{x}_{\mathbf{0}} \in \mathbb{R}^{p}$ is given by :

$$
\widehat{s}\left(\mathbf{x}_{\mathbf{0}}\right)=\sigma^{2}\left(1-\boldsymbol{r}\left(\mathbf{x}_{\mathbf{0}}\right)^{\prime} \boldsymbol{R}^{-1} \boldsymbol{r}\left(\mathbf{x}_{\mathbf{0}}\right)^{\prime}\right)
$$

where $\mathbb{1}_{n}=(1, \ldots, 1)^{\prime} \in \mathbb{R}^{n}, \boldsymbol{R} \in \mathcal{M}_{n \times n}$ is the correlation matrix of the process $(Y)$ at the observation points, $\mathbf{r}\left(\mathbf{x}_{\mathbf{0}}\right) \in \mathcal{M}_{n \times 1}$ the correlation vector between $Y\left(\mathbf{x}_{\mathbf{0}}\right)$ and the random vector $\left(Y\left(\mathbf{x}_{1}\right), \ldots, Y\left(\mathbf{x}_{n}\right)\right)^{\prime}$.

In the following we will focus on the definition of the correlation function $\mathbf{r}$. First, we introduce the anisotropic kernel :

$$
\mathbf{r}(\mathbf{x}-\tilde{\mathbf{x}})=\prod_{j=1}^{p} \rho_{\theta_{j}}\left(\left|x_{j}-\tilde{x}_{j}\right|\right), \boldsymbol{\theta}=\left(\theta_{1}, \ldots, \theta_{p}\right) \in \mathbb{R}_{+}^{p}
$$

where $\rho_{\theta_{j}}$ is a correlation function which only depends on the one dimensional range parameter $\theta_{j}$, see e.g Santner et al. (2003) and Stein (1999). In the following, we denote the model (1) with kernel (4) as $\mathcal{M}_{a}$. The parameters $m, \sigma^{2}$ and $\boldsymbol{\theta}$ are unknown and will be estimated by maximum likelihood.

In our industrial case study the output is supposed to be two times continuously differentiable this is why we use a Matern 5/2 kernel usual in that case, see e.g. Cornford et al. (2002) . This kernel function is defined by :

$$
\forall \theta \in \mathbb{R}^{+}, \forall h \in \mathbb{R}^{+}, \rho_{\theta}(h)=\left(1+\frac{\sqrt{5}|h|}{\theta}+\frac{5 h^{2}}{3 \theta^{2}}\right) \exp \left(-\frac{\sqrt{5}|h|}{\theta}\right) .
$$

Other examples can be found in Rasmussen and Williams (2006). However, anisotropic kernels contain as many parameters as the number of variables $p$. In high dimension the estimation of these parameters becomes unstable. To stabilize the estimation an idea is to reduce the number of range parameters. The extremal case is the isotropic kernel, defined as follows for $\left(\mathbf{x}, \mathbf{x}^{\prime}\right) \in \mathbb{R}^{p}$ :

$$
r(\mathbf{x}-\tilde{\mathbf{x}})=\rho_{\theta}\left(\|\mathbf{x}-\tilde{\mathbf{x}}\|_{2}\right), \theta \in \mathbb{R}_{+}
$$

where $\|.\|_{2}$ is the euclidian norm on $\mathbb{R}^{p}$. In the following, we denote the model (1) with kernel (5) as $\mathcal{M}_{i}$. In model $\mathcal{M}_{i}$, spatial variations are controlled by only one range parameter. If the value of that parameter is small the process $Y$ fluctuates a lot in all directions. On the contrary if the value of $\theta$ is large, the process varies in a very slow way in all directions. That's why, one unique parameter is often too restrictive to characterize the underlying process. In many cases spatial behavior varies from a direction to another. Therefore we introduce in the next section a kernel which is a compromise between the too restrictive isotropic kernel and the too flexible anisotropic one. We call it the isotropic by group kernel. 


\section{$2.2 \quad$ Isotropic by group kernel}

The isotropic by group kernel is defined as follows:

$$
r(\mathbf{x}-\tilde{\mathbf{x}})=\prod_{\ell=1}^{q} \rho_{\theta_{\ell}}\left(\|\mathbf{x}-\tilde{\mathbf{x}}\|_{\mathcal{I}_{\ell}}\right), \boldsymbol{\theta}=\left(\theta_{1}, \ldots, \theta_{q}\right) \in \mathbb{R}_{+}^{q}
$$

where, $q<p$ is the number of groups. Let $\mathcal{I}_{\ell}$ be the set of indexes in the $\ell$ th group with cardinal $\left|\mathcal{I}_{\ell}\right|=p_{\ell}, p_{\ell} \in\{1, \ldots, p\}$ such that $p_{1}+\ldots+p_{q}=p$. We define the norm of the subvector $\left(x_{j}\right)_{j \in \mathcal{I}_{\ell}}$ of the vector $\mathbf{x}=\left(x_{1}, \ldots, x_{p}\right) \in \mathbb{R}^{p}$ by $\|\mathbf{x}\|_{\mathcal{I}_{\ell}}=\sqrt{\left(\sum_{j \in \mathcal{I}_{\ell}} x_{j}^{2}\right)}$. In the following, we denote model (1) with kernel (6) as $\mathcal{M}_{q}$. The anisotropic and isotropic kernel are two particular cases of isotropic by group kernel with respectively $p$ groups $\mathcal{I}_{\ell}=\{\ell\}$ for $\ell=\{1, \ldots, p\}$, and one unique group $\mathcal{I}_{1}=\{1, \ldots, p\}$, ie $\mathcal{M}_{p}=\mathcal{M}_{a}$ and $\mathcal{M}_{1}=\mathcal{M}_{i}$.

In appendix, we simulate with the same seed a Gaussian Process in 2 dimensions with an anisotropic and an isotropic kernel $\left(\mathcal{M}_{a}\right.$ and $\left.\mathcal{M}_{i}\right)$ to visualize the impact of the choice of the kernel.

\section{Methodology}

In this article we propose four strategies to find simultaneously and automatically the number of groups and the composition of each group. In Yi (2009) and Muehlenstaedt et al. (2012) the two proposed methodologies to reduce the number of parameters in kriging start with a fully anisotropic kernel. To gain in robustness in high dimensional problems, in the four procedures we introduce, we always start with an isotropic kernel that depends on a unique range parameter and we finish with an isotropic by group kernel. At each stage we compare different models and we choose the best model under a specific criterion. The model parameters are estimated by maximum likelihood, that is:

$$
\hat{\boldsymbol{\theta}}=\underset{\boldsymbol{\theta}}{\operatorname{argmin}}\left[l\left(\boldsymbol{\theta} ; \mathbf{x}_{1}, \ldots, \mathbf{x}_{n}, \mathbf{y}\right)\right]
$$

where $l=-\log (\mathcal{L})$ and $\mathcal{L}$ is the likelihood function defined by:

$$
\mathcal{L}\left(\boldsymbol{\theta} ; \mathbf{x}_{1}, \ldots, \mathbf{x}_{n}, \mathbf{y}\right)=\frac{1}{\left(2 \pi \hat{\sigma}^{2}\right)^{\frac{n}{2}}|\boldsymbol{R}|^{\frac{1}{2}}} e^{-\left(\mathbf{y}-\hat{m} \mathbb{1}_{n}\right)^{t} \boldsymbol{R}^{-1}\left(\mathbf{y}-\hat{m} \mathbb{1}_{n}\right)}
$$

where $\hat{m}$ and $\hat{\sigma}^{2}$ could be writen as functions of $\boldsymbol{\theta}$ :

$$
\begin{aligned}
\hat{m} & =\left(\mathbb{1}_{n}^{t} \boldsymbol{R}^{-1} \mathbb{1}_{n}\right)^{-1} \mathbb{1}_{n}^{t} \boldsymbol{R}^{-1} \mathbf{y} \\
\hat{\sigma}^{2} & =\frac{1}{n}\left(\mathbf{y}-m \mathbb{1}_{n}\right)^{t} \boldsymbol{R}^{-1}\left(\mathbf{y}-m \mathbb{1}_{n}\right)
\end{aligned}
$$

Equation (7) shows that the estimation of the range parameter $\boldsymbol{\theta}$ is done by minimizing $l$. The minimization of $l$ is very difficult due to the nonlinearity of the objective function. We use a classical numerical method names Limited-memory BFGS to handle optimization. A well known drawback of the optimization algorithms is the dependence of the solution on the initialization point. That's why we create the Algorithm 0 that finds a good initial value for $\boldsymbol{\theta}$ to minimize $l$ and that gives an estimation of $\hat{\boldsymbol{\theta}}$. The principle of Algorithm 0 is to first select 10 vectors of parameters $\boldsymbol{\theta}\left(\boldsymbol{\theta} \in \mathbb{R}^{q}\right)$ that cover space as well as possible and to choose 
the set of parameters, says $\boldsymbol{\theta}_{\text {opt_init }}$, that has the lowest $l$-value. Then, we minimize $l$ using $\boldsymbol{\theta}_{\text {opt_init }}$ as initial point and we obtain the estimation of $\left(\theta_{\ell}\right)_{\ell=1, \ldots, q}$, denoted by $\boldsymbol{\theta}_{\text {opt }}$.

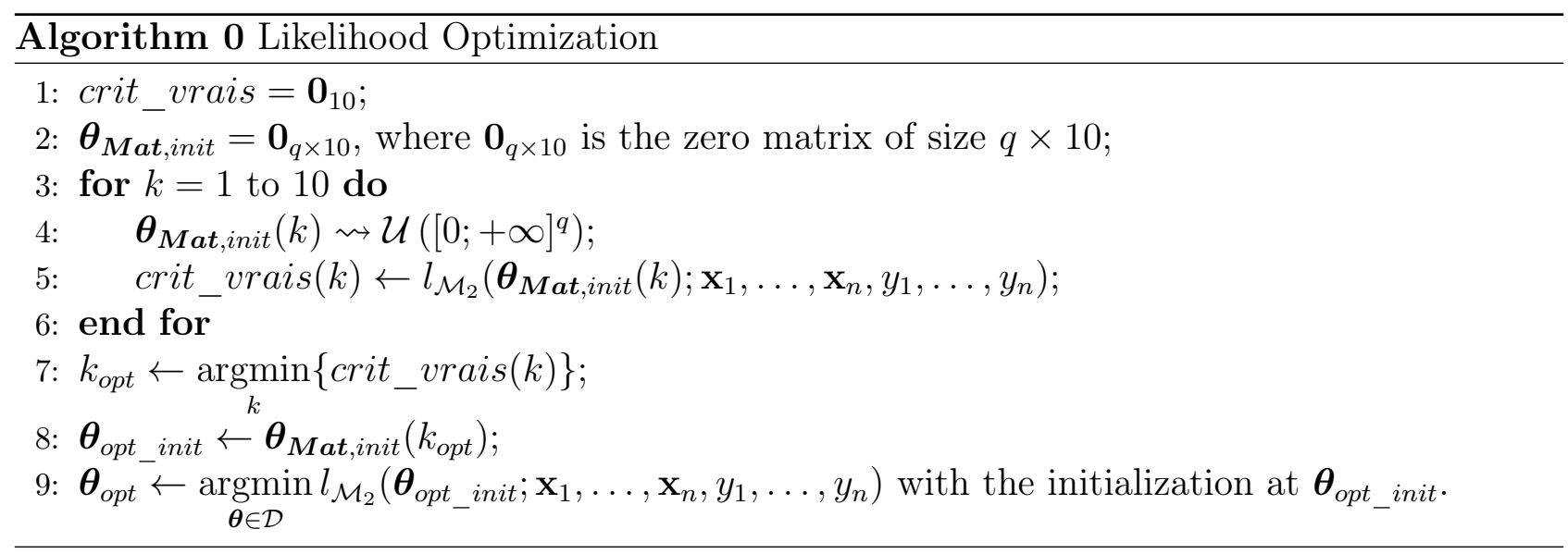

As we estimate parameters by maximum likelihood, it could then be natural to select the best model under likelihood considerations. However the likelihood is an increasing function of the number of parameters. As a trade off between estimation qualities and sparsity, a penalized likelihood criterion is preferred. In our work, we consider the BIC criterion (see e.g. Schwarz (1978)), that is for a model $\mathcal{M}_{q}$ with $q$ parameters to estimate $\boldsymbol{\theta}_{q}$ :

$$
B I C\left(\boldsymbol{\theta}_{q}, \mathcal{M}_{q}\right)=l_{\mathcal{M}_{q}}\left(\boldsymbol{\theta}_{q} ; \mathbf{x}_{1}, \ldots, \mathbf{x}_{n}, \mathbf{y}\right)+q \log (n) .
$$

In the four strategies, the best model corresponds to the one minimizing the BIC criterion. Another usual criterion for selecting the best model is based on predictive considerations, called the cross-validation criterion. But this criterion is very time consuming as the estimation in the context of high dimensional data. So we decide to not consider this criterion.

\subsection{Algorithm 1}

\section{General loop:}

At each step $k$, if the previous model is composed of $k-1$ groups, the next one will have $k$ groups. Let $\mathcal{M}_{k-1}$ be the model at step $k-1$, we note $\mathcal{I}_{1}=\left\{i_{1,1}, \ldots, i_{1, p_{1}}\right\} ; \ldots ; \mathcal{I}_{k}=$ $\left\{i_{k-1,1}, \ldots, i_{k-1, p_{k-1}}\right\}$, with $p_{1}+\ldots+p_{k-1}=p$, the sets of indexes of the variables included in each group. One of these $k-1$ groups is then divided into two groups. We have $J_{k}$ ways of choosing this group, with $J_{k}=\sum_{\ell=1}^{k-1} J_{k, \ell}$ and $J_{k, \ell}$ is the number of ways of dividing into two groups the set of indexes $\mathcal{I}_{\ell}, \ell=1, \ldots, k-1$. We note $\mathcal{M}_{k}^{1}, \ldots, \mathcal{M}_{k}^{J_{k}}$ the $J_{k}$ new models. And among these $J_{k}$ models we choose the one minimizing the BIC, says $\mathcal{M}_{k}^{\ell_{\text {opt }}}$.

\section{Algorithm 1:}

We start with the estimation of the full isotropic model, noted $\mathcal{M}_{1}$. We follow the previous procedure and we obtain $J_{2}$ models noted $\mathcal{M}_{2}^{\ell}, \ell=1, \ldots, J_{2}$. We estimate the parameter $\boldsymbol{\theta}$ of each model and we keep the model $\mathcal{M}_{2}^{\ell \text { opt }}$ that minimizes the BIC. If the BIC of the model $\mathcal{M}_{1}$ is smallest than the one of $\mathcal{M}_{2}$, we stop the algorithm and we choose $\mathcal{M}_{1}$ as the best model. Otherwise, we set down $\mathcal{M}_{2}=\mathcal{M}_{2}^{\ell_{\text {opt }}}$ and we build the models $\mathcal{M}_{3}^{\ell} l=1, \ldots, J_{3}$ and so forth at step $4,5, \ldots$. We stop the algorithm when $\mathcal{M}_{k}$ has a larger BIC than $\mathcal{M}_{k}$. At the end of the algorithm, we obtain the model $\mathcal{M}_{q}$ that minimizes the BIC. 


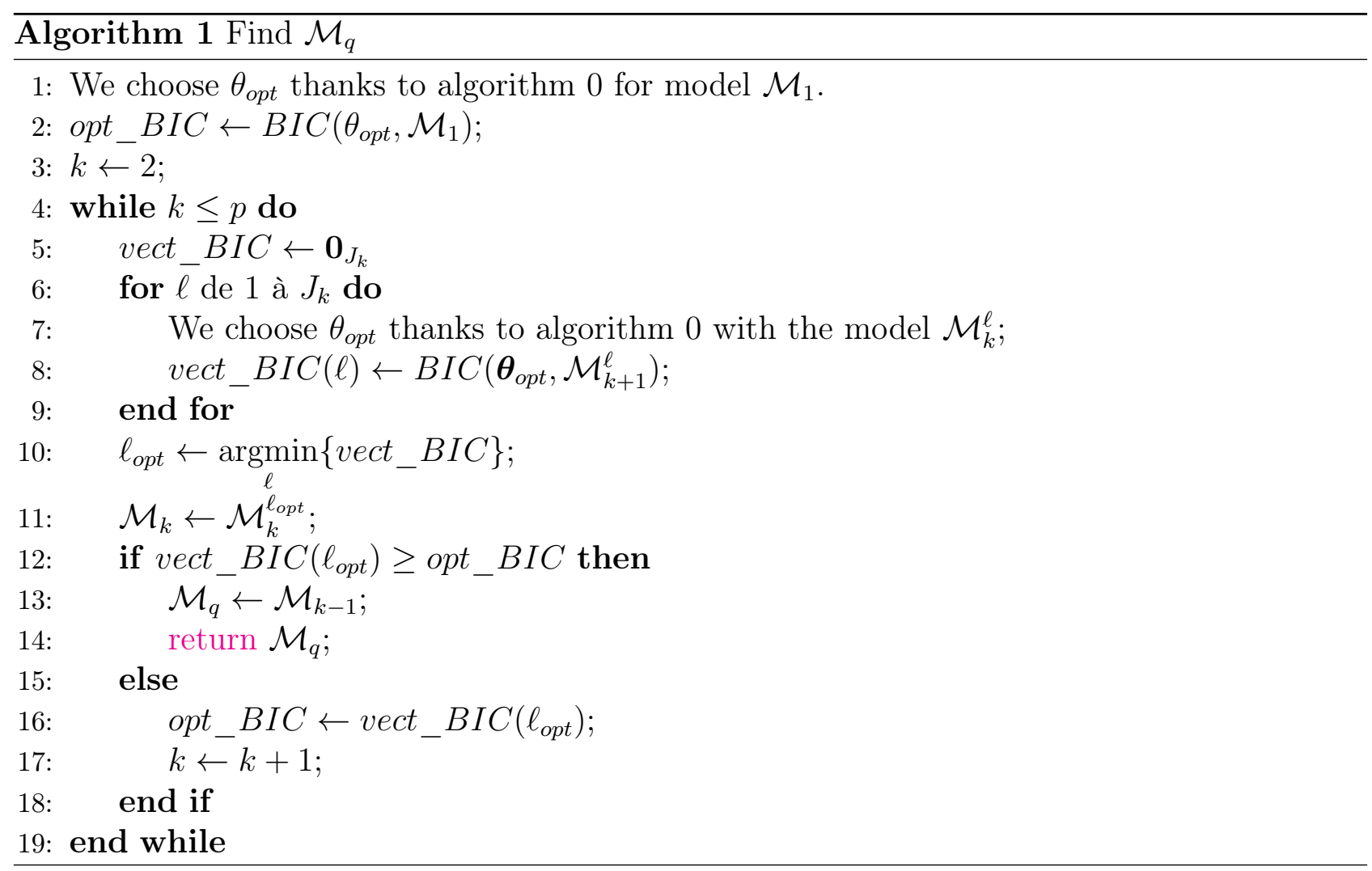

Algorithm 1 is a quasi-exhaustive algorithm since at each step it browses all the possibilities to divide. But the calculation time grows really fast with the number of inputs $p$. For example, the first separation, that consists in separating one group in two, estimates 500 models for $p=10$ and 524287 for $p=20$. That's why we propose several alternative algorithms that take much less time.

\subsection{Algorithm 2}

We propose a faster algorithm close to the forward Algorithm W introduced by Welch et al. (1992) that is described in appendix. The direction of Algorithm 2 is both because it has a backward step contrary to Algorithm W. Algorithm W is included in Algorithm 2.

We note $\mathcal{M}_{s_{k-1}}$ the model at step $k-1$ and $s_{k-1}$ the number of groups. Let $\mathcal{I}_{1}=\left\{i_{1,1}, \ldots, i_{1, p_{1}}\right\}$ $; \ldots ; \mathcal{I}_{s_{k-1}}=\left\{i_{s_{k-1}, 1}, \ldots, i_{s_{k-1}, p_{s_{k-1}}}\right\}$, with $p_{1}+\ldots+p_{s_{k-1}}=p$, the variables' indexes of each group.

\section{Checking step:}

At step $k$, there are 2 possibilities :

(i) If we have grouped some variables at the step $k-1$ or if the current step is $k<3$ (aggregation is not allowed before $k=3$ ), we create an additional group by taking an index $i_{1, m} \in \mathcal{I}_{1}, m=1, \ldots, p_{1}$ out of the group 1 . We have $J_{k}=J_{k}^{+}=p_{1}$ possibilities and so we build $J_{k}$ models.

(ii) If we have separated at the step $k-1$ and $k \geq 3$, we have 2 possibilities. The first one consists in taking an index $i_{1, s} \in \mathcal{I}_{1}, s=1, \ldots, p_{1}$ out of the first group, we have $J_{k}^{+}=p_{1}$ possible indexes. The second consists in grouping the last taken out variable $i_{s_{k-1}, 1}\left(p_{s_{k-1}}=1\right)$ with an other group, we have $J_{k}^{-}=s_{k-1}-2$ possibilities. Then we build a total of $J_{k}=J_{k}^{+}+J_{k}^{-}=p_{1}+s_{k-1}-2$ models. 
In the two cases, we create $J_{k}$ models noted $\mathcal{M}_{s_{k_{1}}}^{1}, \ldots, \mathcal{M}_{s_{J_{k}}}^{J_{k}}$.

\section{General loop:}

We follow the general procedure in introducing a boolean that represents the choice at step $k-1$ of grouping or separating. Then the value of the boolean will be TRUE if we grouped at the previous step and FALSE if not.

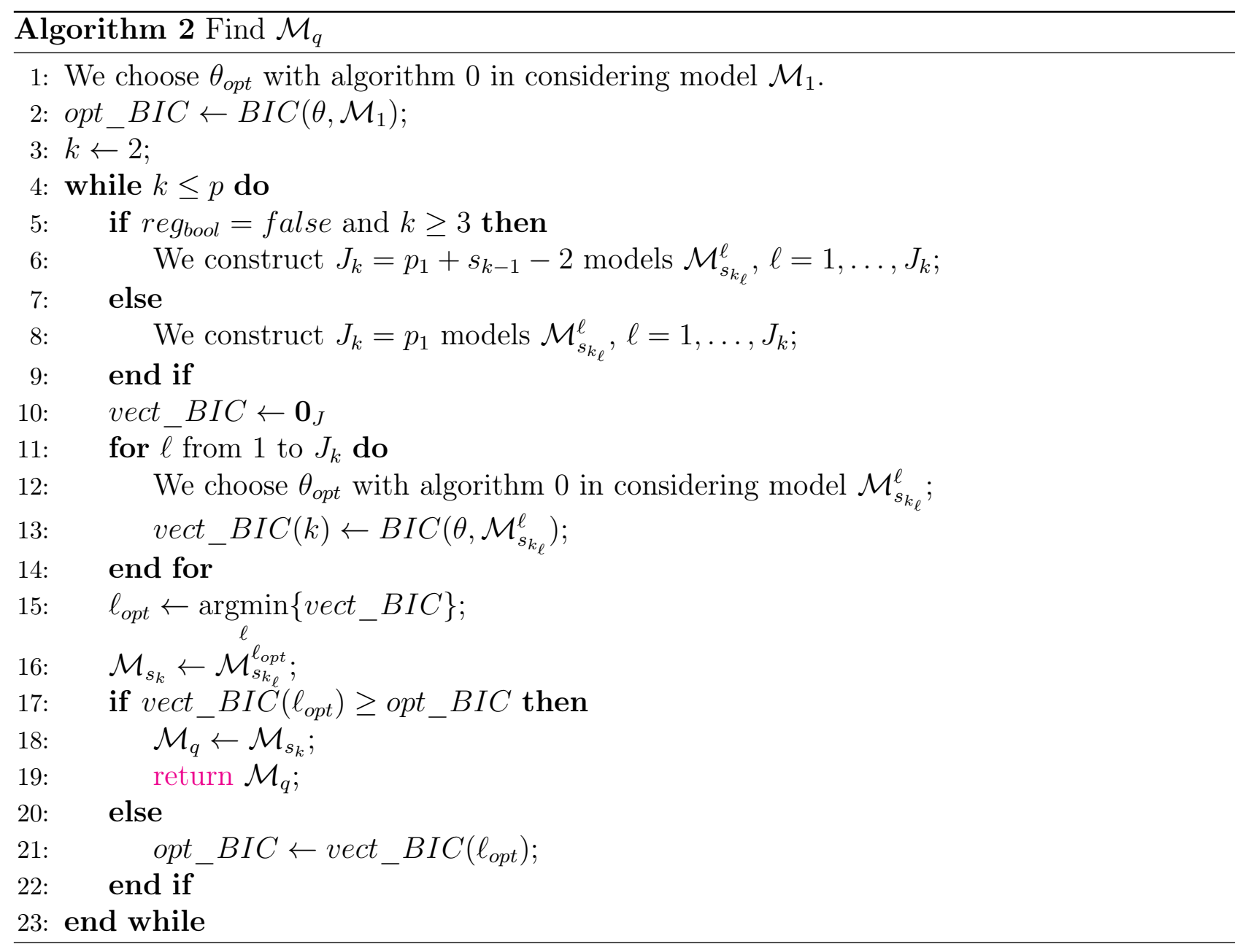

Algorithm 2 divides more than Algorithm 1 and browses less possibilities. This algorithm is largely identical to Algorithm W introduced by Welch Welch et al. (1992) except that we add a backward step.

\subsection{Algorithm 3}

Because Algorithm 2 generates a number of clusters often too high, we propose to add a backward step at the end of the procedure. This step naturally reduces the complexity of the model.

\section{General loop:}

Let $\mathcal{M}_{q}$ the best model found by Algorithm 2, such that $\mathcal{I}_{1}=\left\{i_{1,1}, \ldots, i_{1, p_{1}}\right\} ; \ldots ; \mathcal{I}_{q}=$ $\left\{i_{q, 1} \ldots, i_{q, p_{q}}\right\}$, with $p_{1}+\ldots+p_{q}=p$ the indexes of variables in each groups. We build a new model noted $\mathcal{M}_{\text {clust }}$ with a clustering method that groups variables with close ranges (see Everitt et al. (2011)). However, in the isotropic by group kernel the ranges are not comparable since they are linked to variable sets of different sizes. So we introduce the following kernel , 


\begin{tabular}{l|llll|l}
\hline Algorithms & alg1 & alg2 & alg3 & alg4 & algW \\
\hline Strategy & Quasi & Separate & $\begin{array}{l}\text { Separate } \\
\text { + Cluster }\end{array}$ & Separate & Separate \\
& exhaustive & & $\begin{array}{l}\text { + Cluster } \\
\text { at the end }\end{array}$ & at each step & \\
Direction & Forward & Both & Both & Both & Forward \\
\hline
\end{tabular}

Table 1: Strategy and direction of the 4 algorithms and algorithm proposed by Welch et al. (1992)

called the product kernel, where range parameters can be compared :

$$
r_{\boldsymbol{\theta}}(\mathbf{x}-\tilde{\mathbf{x}})=\prod_{\ell=1}^{q} \prod_{j=1}^{p_{\ell}} \rho_{\theta_{\ell}}\left(\left|x_{j}-\tilde{x}_{j}\right|\right)
$$

It is a tensor product kernel with some equal range parameters. In the following, we denote this model $\mathcal{M}_{q}^{\text {prod }}$. Then we estimate the model $\mathcal{M}_{q}^{\text {prod }}$ with the same groups than the model $\mathcal{M}_{q}$. We build the model $\mathcal{M}_{\text {clust }}$ with a clustering method that groups variables with close range parameter values. Then, if the BIC of the model $\mathcal{M}_{\text {clust }}$ is smaller that the one of $\mathcal{M}_{q}$ we set down $\mathcal{M}_{q}=\mathcal{M}_{\text {clust }}$. We replace the step 19 in Algorithm 2 by:

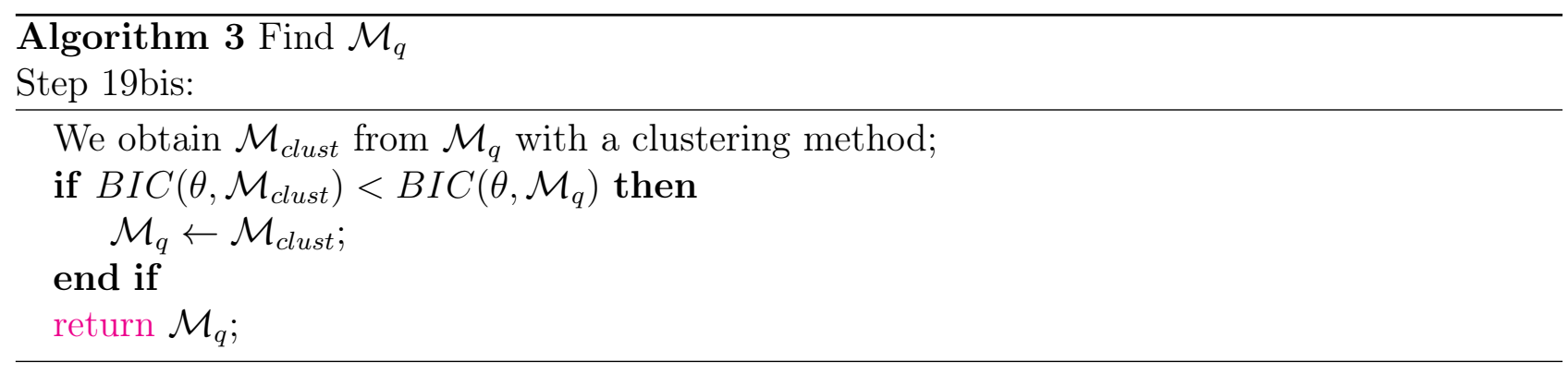

\subsection{Algorithm 4}

Algorithm 4 combines Algorithm 2 with a clustering method at each step $k$ of the algorithm. General loop:

At each step $k$ we compute $\mathcal{M}_{s_{k}}$ by Algorithm 2. Then, with the same groups composition we estimate $\mathcal{M}_{s_{k}}^{\text {prod }}$ to which we apply a classification step to produce $\mathcal{M}_{k, \text { clust }}$. If the BIC of model $\mathcal{M}_{k, \text { clust }}$ is smaller that the one of $\mathcal{M}_{s_{k}}$ we set down $\mathcal{M}_{s_{k}}=\mathcal{M}_{k, \text { clust }}$. We replace the step 16 in Algorithm 2 by:

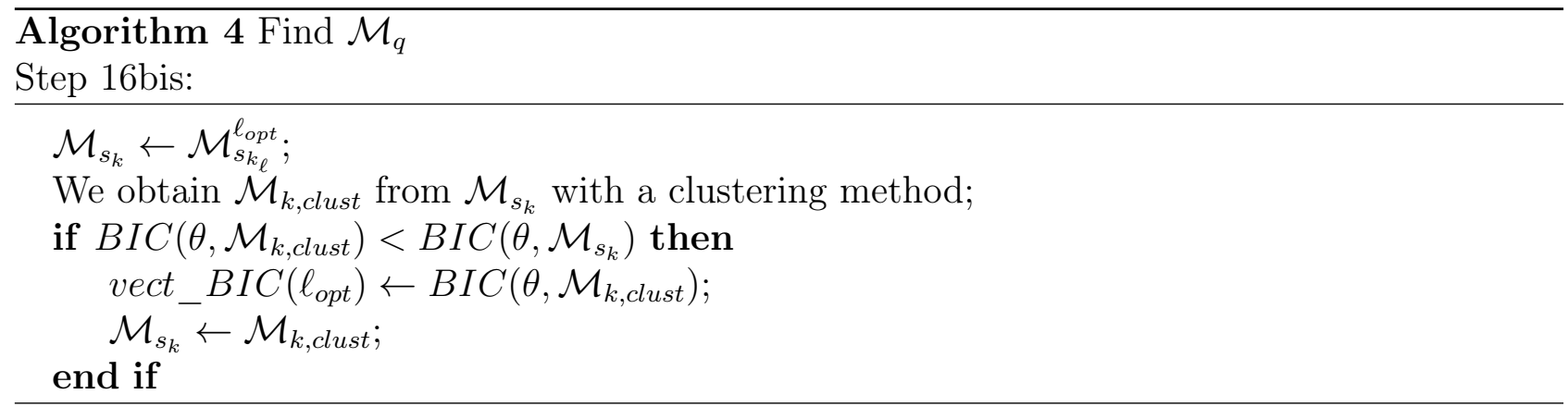

\subsection{Conclusion and summary}

The Table 1 summarizes the general characteristics of the algorithms. 


\section{Application}

\subsection{Analytical examples}

In this section we compare the algorithms and we observe the behavior of the best one according to the number of observations and to the nugget effect.

\subsubsection{Algorithms' Comparison}

We simulate a Gaussian process with $p=8$ parameters, the simulated model is :

$$
Y(\mathbf{x})=1+\epsilon(\mathbf{x})
$$

where $\epsilon$ is a Gaussian process with a standard deviation $\sigma^{2}=1$ and an isotropic by group kernel:

$$
r_{\boldsymbol{\theta}}(\mathbf{x}-\tilde{\mathbf{x}})=\prod_{\ell=1}^{3} \rho_{\theta_{\ell}}\left(\|x-\tilde{x}\|_{\mathcal{I}_{\ell}}\right)
$$

where $\mathcal{I}_{\ell}=\{1\}, \mathcal{I}_{2}=\{2,3\}, \mathcal{I}_{3}=\{4,5,6,7,8\}$ and $\rho_{\theta}$ is a Matern5_2. $\boldsymbol{\theta}=(0.5,0.9,0.8)$. The learning set is an optimized Latin Hypercube with $n$ observations and the test set is a Sobol sequences of 1000 points.

We simulate 100 different trajectories of the model with $n=800$ points of observations. For each trajectory we estimate the model with the algorithms. Table 2 shows that each algorithm finds the correct number and composition of groups for the 100 trajectories except Algorithm W. This result is not surprising because Algorithm W separates variable by variable and cannot propose several groups with more than one variable. Among the four algorithms, Table

\begin{tabular}{c|cc} 
& good groups & too separate \\
\hline alg1 & 100 & 0 \\
alg2 & 100 & 0 \\
alg3 & 100 & 0 \\
$\operatorname{alg} 4$ & 100 & 0 \\
algW & 0 & 100
\end{tabular}

Table 2: Gathering errors done by the algorithms for 100 trajectories with one learning set of size 800 .

3 shows that Algorithm 4 is the fastest, it takes 1 h21 to find the best model. In the next sections we only use Algorithm 4.

\begin{tabular}{l|c} 
alg & Calculation time by processor \\
\hline alg1 & $6 \mathrm{~h} 38$ \\
alg2 & $1 \mathrm{~h} 52$ \\
$\operatorname{alg} 3$ & $1 \mathrm{~h} 50$ \\
$\operatorname{alg} 4$ & $1 \mathrm{~h} 21$ \\
$\operatorname{algW}$ & $1 \mathrm{~h} 20$
\end{tabular}

Table 3: Calculation time done by the four algorithms for 100 trajectories with one learning set of size 800 . 


\subsubsection{Evolution of the prediction quality with the learning set size}

We test nine learning set sizes : $n=\{80,280,480,680,880,1080,1280,1480,1680\}$. For each size we simulate one learning set and 25 trajectories. For the high size, the algorithm could not converge due to the bad conditioning of the covariance matrix. There is too many observations points for the kriging. For a learning set of size 80, the algorithm makes lot of bad gathering

\begin{tabular}{l|ccc|c} 
Sample size & good groups $(\%)$ & bad gathering $(\%)$ & too separate $(\%)$ & Simulations \\
\hline 80 & 16 & 84 & 0 & 25 \\
280 & 80 & 4 & 16 & 25 \\
480 & 100 & 0 & 0 & 25 \\
680 & 100 & 0 & 0 & 25 \\
880 & 100 & 0 & 0 & 25 \\
1080 & 100 & 0 & 0 & 23 \\
1280 & 100 & 0 & 0 & 20 \\
1480 & 100 & 0 & 0 & 17 \\
1680 & 100 & 0 & 0 & 12
\end{tabular}

Table 4: Gathering errors done by the Algorithm 4 for 25 trajectories with different learning set size $\{80,280,480,680,880,1080,1280\}$

(see Table 4). These errors are serious because they cause a loss of prediction quality. Figure

Time to find the model with algorithm $\mathbf{4}$ for one simulation

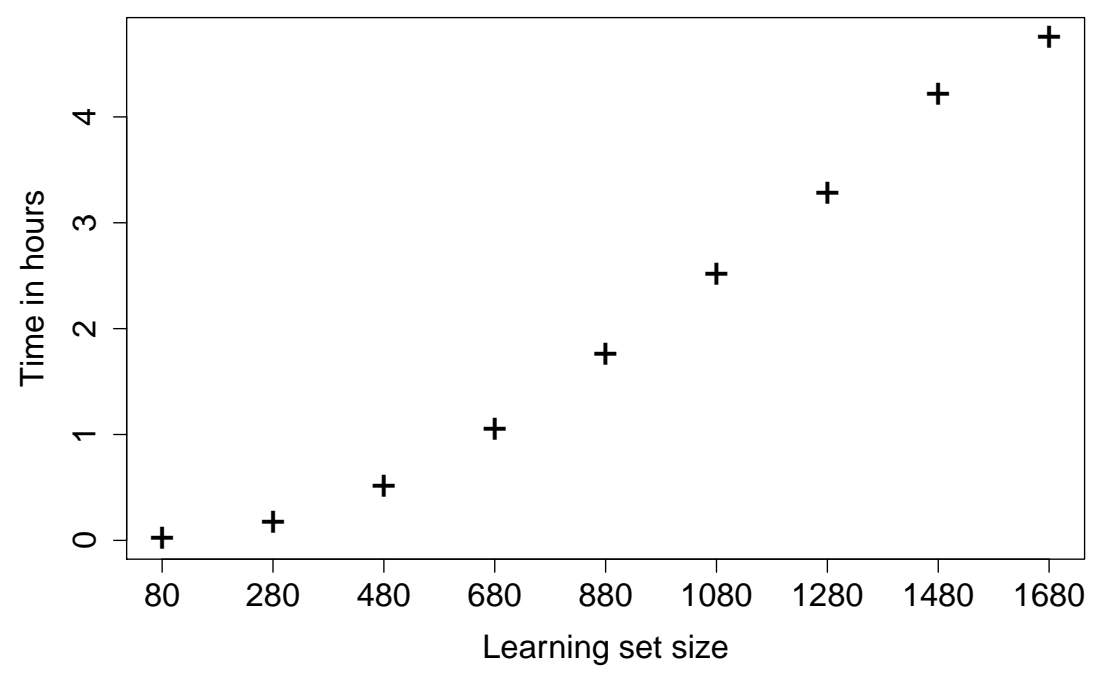

Figure 1: Time in hours to find the best model with the Algorithm 4. 25 trajectories are simulated for the each size $\{80,280,480,680,880,1080,1280,1480,1680\}$.

1 shows that the estimation time grows with the number of experiments. For a size of 1280 the estimation takes more than 4 hours (see Table 3 ). The prediction quality also increases with the number of experiments. From $n=680$, the prediction quality becomes very good (see Figure 2).

Whatever the size of the learning set kriging with an Isotropic by group kernel stays the best (see Figure 3). In addition, with a low number of points (80), we notice that the anisotropic give a completely wrong prediction in $20 \%$ of simulations. Contrary to the isotropic by group and isotropic stay efficient. 

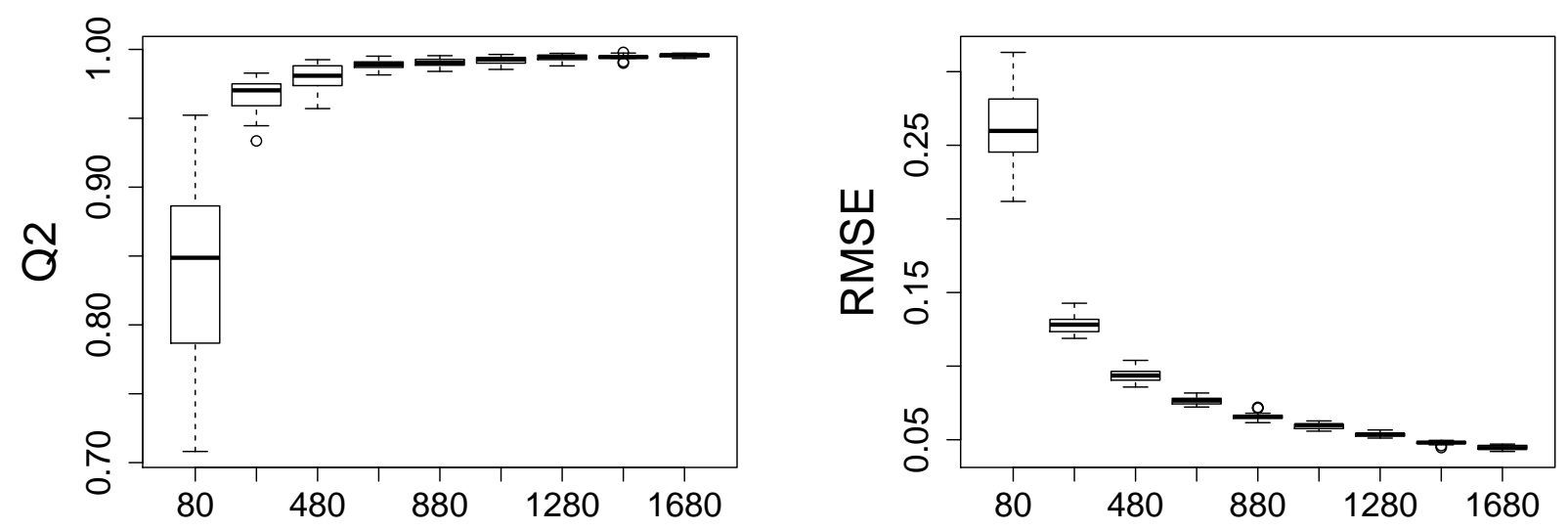

Figure 2: Prediction quality of the model with an isotropic by group kernel found by Algorithm 4 according to the sample size. 25 trajectories are simulated for each size $\{80,280,480,680,880,1080,1280,1480,1680\}$. The criteria of prediction quality are Q2 and RMSE.

\subsubsection{Evolution of the prediction quality with an error on simulation parameters}

We simulated the model :

$$
Y(\mathbf{x})=1+\epsilon(\mathbf{x})
$$

where $\epsilon$ is a Gaussian process with standard deviation $\sigma^{2}=1$ and an anisotropic kernel:

$$
r_{\boldsymbol{\theta}}(\mathbf{x}-\tilde{\mathbf{x}})=\prod_{j=1}^{8} \rho_{\theta_{j}^{*}}\left(\left|x_{j}-\tilde{x}_{j}\right|\right)
$$

where $\rho_{\theta}$ is a Matern $5 \_2$ and $\boldsymbol{\theta}=(0.5,0.9,0.9,0.8,0.8,0.8)$. We simulate the range parameters with an error $\xi=(0,2,5,10,15,40)$ such that $\theta_{j}^{*} \sim \mathcal{U}\left(\left[\theta_{j}-\frac{\xi_{k}}{100} \theta_{j} ; \theta_{j}-\frac{\xi_{k}}{100} \theta_{j}\right]\right), k=1, \ldots, 6$ and $j=1, \ldots, 8$. The learning set is an optimized Latin Hypercube with $n=400$ observations and the test set is a Sobol sequence of 1000 points. For each value of $\xi$ we simulate 25 trajectories.

Adding an error to range parameters doesn't influence the prediction quality of $\mathcal{M}_{q}$ and it stays the best compared to $\mathcal{M}_{a}$ and $\mathcal{M}_{i}$, see Figure 4 .

\subsubsection{Evolution of the prediction quality with a nugget effect}

We take the same Gaussian process as previously but with a nugget effect:

$$
r_{\boldsymbol{\theta}}(\mathbf{x}-\tilde{\mathbf{x}})=\prod_{j=1}^{p} \rho_{\theta_{j}}\left(\left|x_{j}-\tilde{x}_{j}\right|\right)+\tau^{2} \delta_{0}(\mathbf{x}-\tilde{\mathbf{x}})
$$

where $\rho_{\theta}$ is a Matern5_2. $\boldsymbol{\theta}=(0.5,0.9,0.9,0.8,0.8,0.8)$. The learning set is an optimized Latin Hypercube with $n$ observations and the test set is a Sobol sequence of 1000 points.

$$
\delta_{0}(\mathbf{x}-\tilde{\mathbf{x}})= \begin{cases}1 & \text { if } \mathbf{x}=\tilde{\mathbf{x}} \\ 0 & \text { if not }\end{cases}
$$

We test 6 different values of nugget effect $\tau=(0,0.02,0.03,0.05,0.1,0.3)$. 

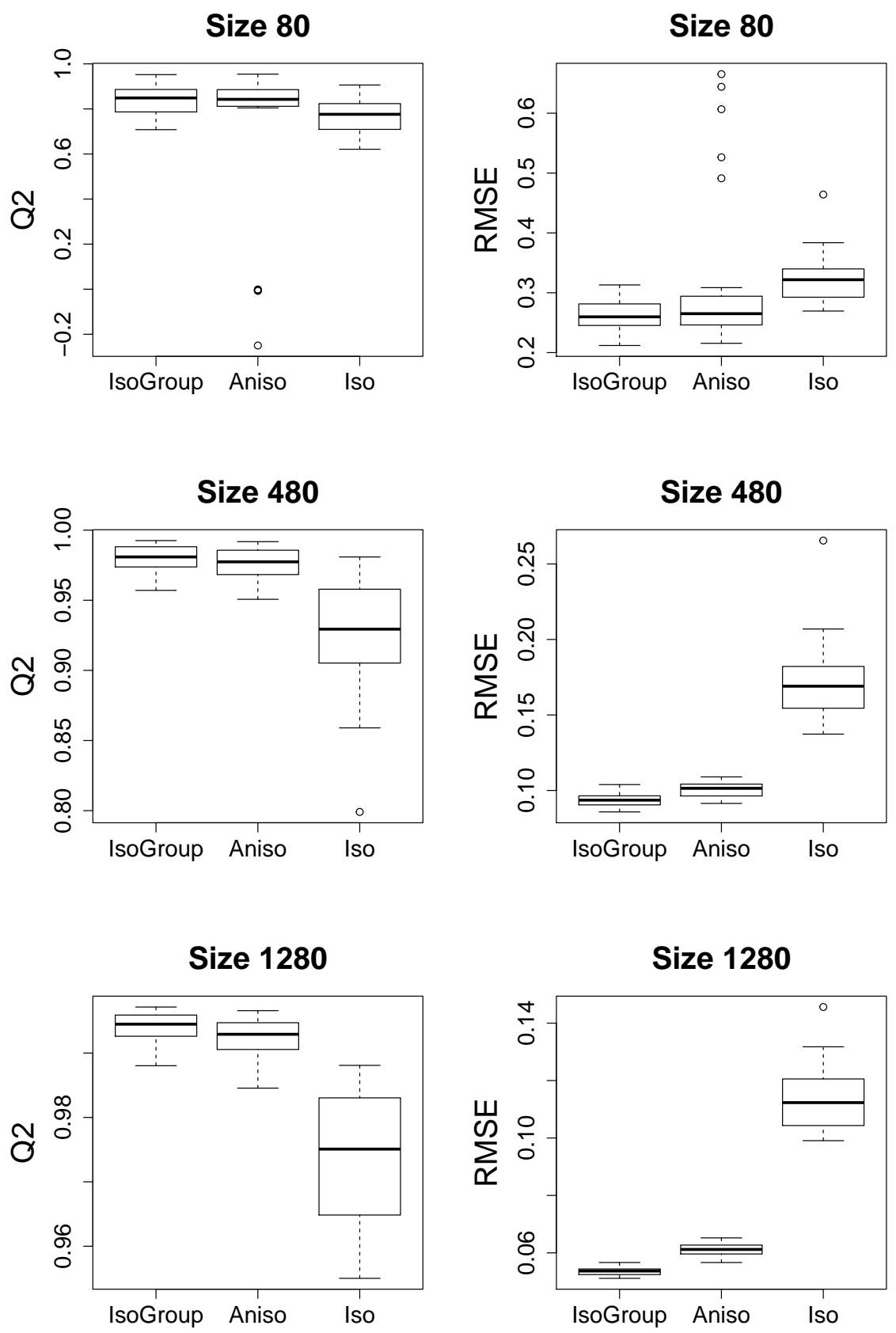

Figure 3: Prediction quality of the model with an isotropic by group kernel found by Algorithm 4 on the left, anisotropic kernel in the middle and isotropic kernel on the right according to the sample size. 25 trajectories are simulated for each learning set size $\{80,480,1280\}$. The criteria of prediction quality are Q2 and RMSE.

Figure 5 shows a loss of prediction quality for each model. Isotropic by group kernel is not a good solution in the presence of a nugget effect. It stays comparable to the other models (anisotropic and isotropic) estimated a without nugget effect. In this context, whatever the kernel, allowing the estimation of a nugget effect is the correct solution. 

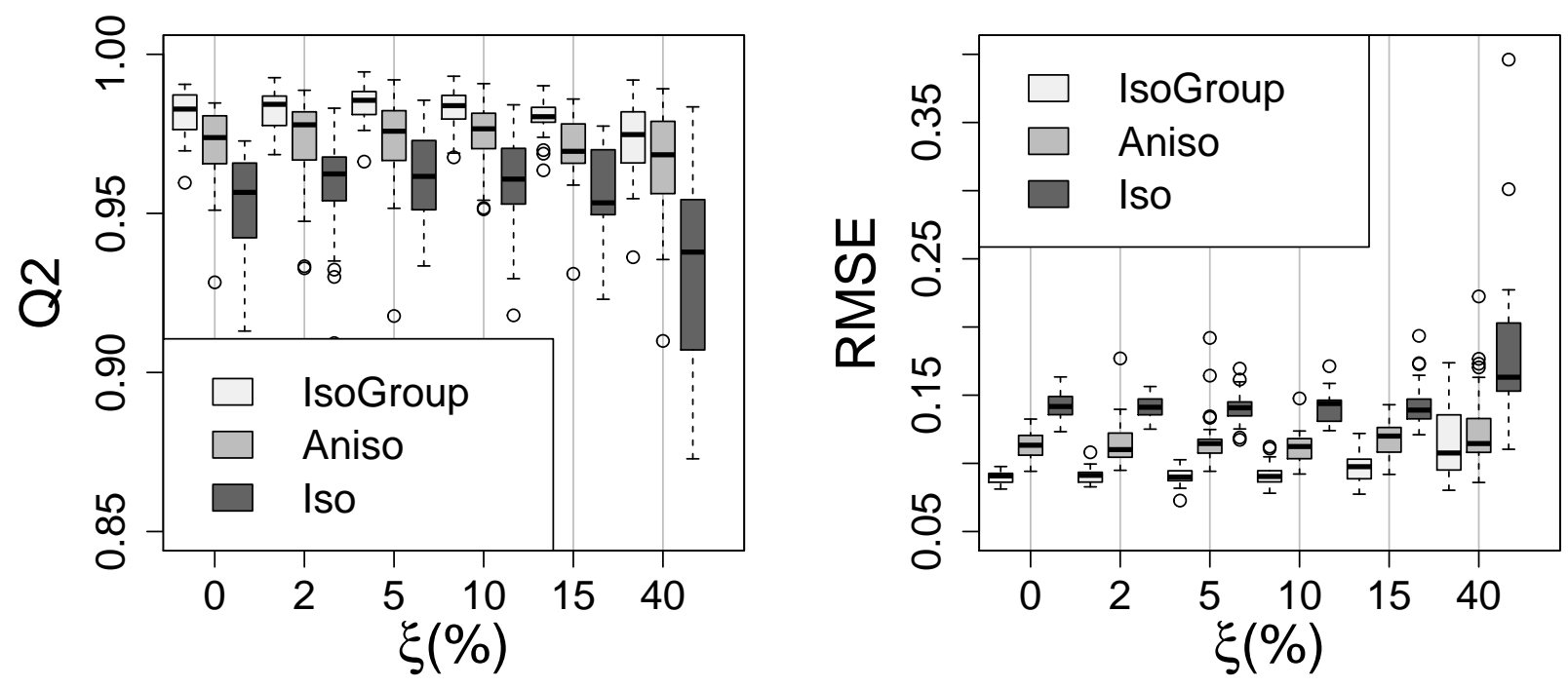

Figure 4: Prediction quality of the model with an isotropic by group kernel found by Algorithm 4 (IsoGroup) anisotropic kernel (Aniso) and isotropic kernel (Iso) according to an error added on the range parameters in the simulated model. 25 trajectories are simulated for a learning set of size 400. The prediction quality criteria are Q2 and RMSE.
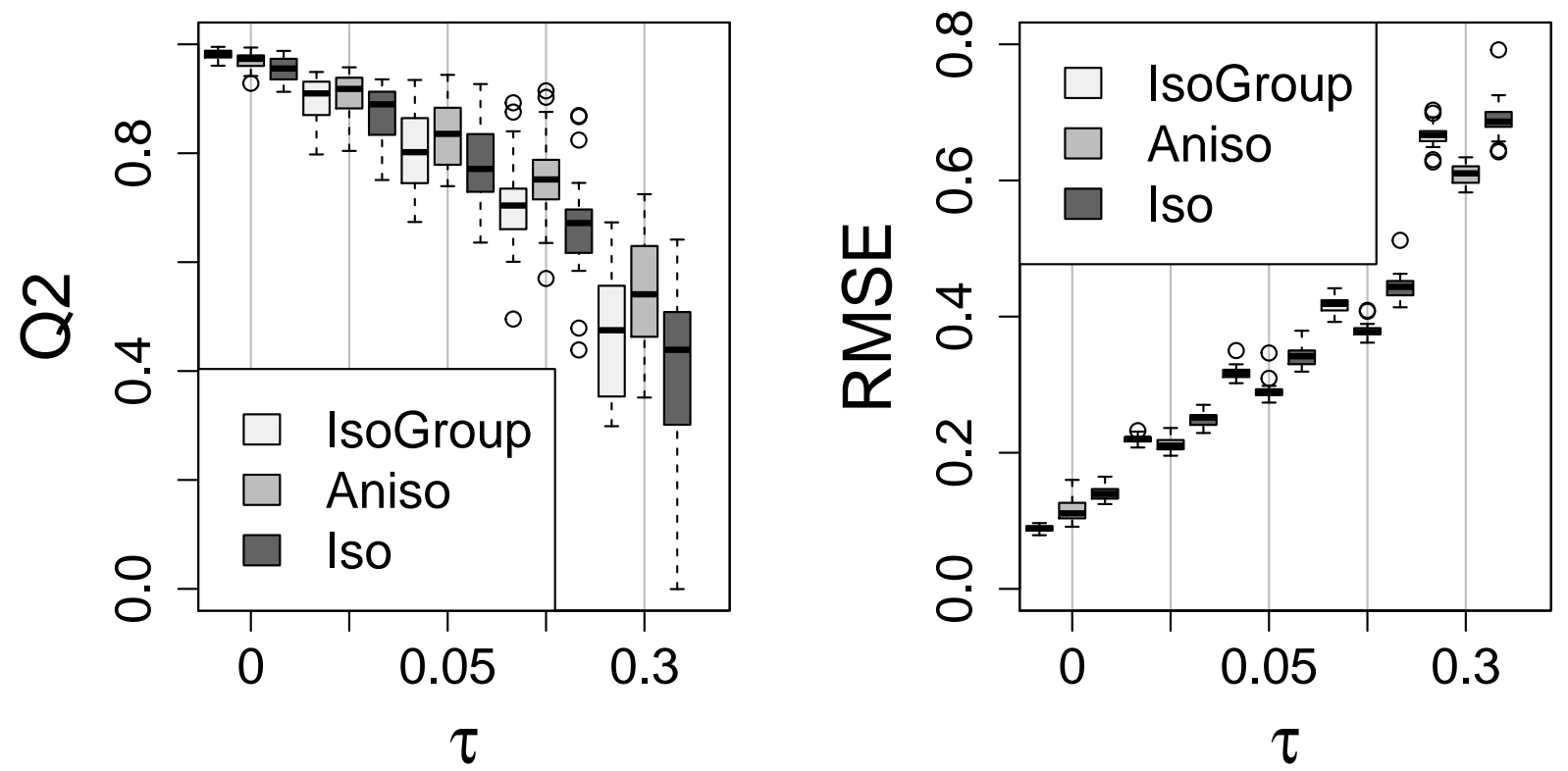

Figure 5: Prediction quality of the model with an isotropic by group kernel found by Algorithm 4 (IsoGroup) anisotropic kernel (Aniso) and isotropic kernel (Iso) according to the value of a nugget effect added in the simulated model. 25 trajectories are simulated for a learning set of size $\{160\}$. The prediction quality criteria are Q2 and RMSE.

\subsection{Test function}

To motivate the construction of our kriging model, let us consider the function::

$$
f(\mathbf{x})=\prod_{j \pm 4}^{15} \frac{4 x_{j}+a_{i}}{1+a_{i}}
$$


with $a^{j}=(0,1,1,4.5,10,10,99,99,99,99,99,99,99,99,99) . y_{1}, \ldots, y_{n}$ are the observations such that $y_{i}=f\left(\mathbf{x}_{i}\right)$ where $f$ is the function and $\mathbf{x}_{i} \in[0 ; 1]^{15}$. Considering $250 \simeq 17 \times 15$ runs of the function, we aim to construct a predictive model based on kriging, with three different kernels: isotropic $\left(\mathcal{M}_{i}\right)$, anisotropic $\left(\mathcal{M}_{a}\right)$ and isotropic by group $\left(\mathcal{M}_{q}\right.$ is found automatically by the Algorithm 4) based on tree different correlation functions:

- Anisotropic $\left(\mathcal{M}_{a}\right)$ :

$$
\mathbf{r}_{\boldsymbol{\theta}}(\mathbf{x}-\tilde{\mathbf{x}})=\prod_{j=1}^{15} \rho_{\theta_{j}}\left(\left|x_{j}-\tilde{x}_{j}\right|\right)
$$

- Isotropic $\left(\mathcal{M}_{i}\right)$ :

$$
\mathbf{r}_{\boldsymbol{\theta}}(\mathbf{x}-\tilde{\mathbf{x}})=\rho_{\theta}\left(\|\mathbf{x}-\tilde{\mathbf{x}}\|_{2}\right)
$$

- Isotropic by group $\left(\mathcal{M}_{4}\right)$ :

$$
\mathbf{r}_{\boldsymbol{\theta}}(\mathbf{x}-\tilde{\mathbf{x}})=\rho_{\theta_{1}}\left(\sqrt{\sum_{j=1}^{3}\left(x_{j}-\tilde{x}_{j}\right)^{2}}\right) \times \rho_{\theta_{2}}\left(\left|x_{4}-\tilde{x}_{4}\right|\right) \times \rho_{\theta_{3}}\left(\sqrt{\sum_{j=5}^{6}\left(x_{j}-\tilde{x}_{j}\right)^{2}}\right) \times \rho_{\theta_{4}}\left(\sqrt{\sum_{j=7}^{15}\left(x_{j}-\tilde{x}_{j}\right)^{2}}\right)
$$

The parameters $m, \sigma^{2}$ and $\boldsymbol{\theta}$ of different models have to be estimated from the sample. $\rho_{\theta}$ is the Matern 5_2 correlation function. In Figure 6, we compare the predictive power of
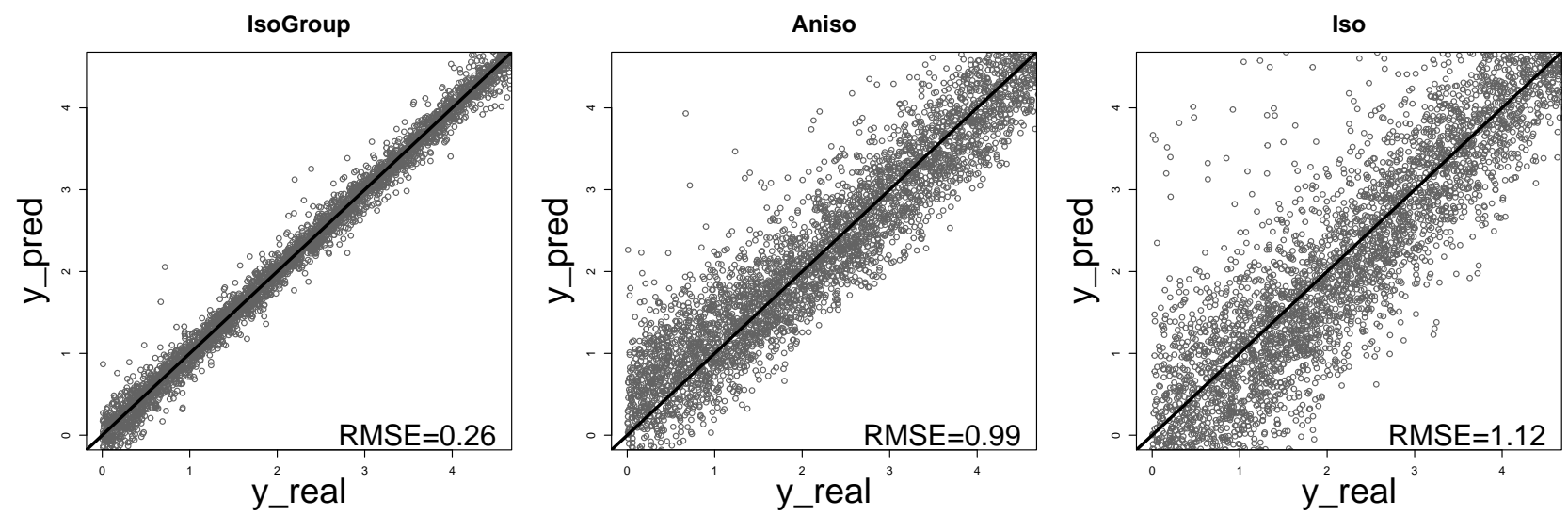

Figure 6: Kriging prediction plots for the function : isotropic by group kernel(left), anisotropic kernel(middle), isotropic kernel (right).

three models : kriging with an isotropic kernel is poor, kriging with an isotropic by group kernel improves prediction power compared to kriging with an anisotropic kernel. Model $\mathcal{M}_{4}$ estimates 4 range parameters instead of 15 for model $\mathcal{M}_{a}$ and 1 for model $\mathcal{M}_{i}$. The estimates of $\boldsymbol{\theta}, m$ and $\sigma^{2}$ are in Table 5 . Table 5 shows that the range parameter groups correspond to close values of $a$ in the Sobol function. Thus, the isotropic by group kernel allows a rise of the number of inputs.

\section{Conclusion}

In high dimension, kriging model with classical kernels provide poor predictions of the response. Yet, with a sparse kernel, predictions are much better. After a study of the algorithms' behavior on their prediction quality and their time of estimation. It results that Algorithm 4 is the 
Isotropic by group kernel

\begin{tabular}{lllllll}
\hline$\theta_{1}\left(x_{1}, x_{3}\right)$ & $\theta_{2}\left(x_{2}\right)$ & $\theta_{3}\left(x_{4}\right)$ & $\theta_{4}\left(x_{5}, x_{6}\right)$ & $\theta_{5}\left(x_{7}, \ldots, x_{15}\right)$ & $\mathrm{m}$ & $\sigma^{2}$ \\
1.53 & 1.8 & 2.57 & 4.6 & 42.12 & 1.78 & 207.06 \\
\hline
\end{tabular}

Anisotropic kernel

\begin{tabular}{lllllllll}
\hline$\theta_{1}$ & $\theta_{2}$ & $\theta_{3}$ & $\theta_{4}$ & $\theta_{5}$ & $\theta_{6}$ & $\theta_{7}$ & $\theta_{8}$ & $\theta_{9}$ \\
0.63 & 0.91 & 0.87 & 1.42 & 2 & 2 & 1.99 & 1.99 & 2 \\
$\theta_{10}$ & $\theta_{11}$ & $\theta_{12}$ & $\theta_{13}$ & $\theta_{14}$ & $\theta_{15}$ & $\mathrm{~m}$ & $\sigma^{2}$ & \\
2 & 2 & 1.99 & 1.99 & 1.99 & 1.99 & 6.67 & 9.78 & \\
\hline
\end{tabular}

\begin{tabular}{|c|c|c|}
\hline \multicolumn{3}{|c|}{ Isotropic kernel } \\
\hline $\bar{\theta}$ & & \\
\hline 3.2 & 6.35 & 177.49 \\
\hline
\end{tabular}

Table 5: Kriging parameters for an isotropic by group kernel (top), an anisotropic kernel (middle) and an isotropic kernel(bottom). The learning set is an optimized Latin Hypercube of size 250. The test set is a Sobol sequence of 8000 points.

most efficient. The estimation time does not grow too fast with the number of experiments and the quality of prediction is always the best. The prediction quality increases with the number of experiments. Adding an error on the simulate range parameters influences the groups composition but not the prediction quality of the isotropic by group model. On the other hand adding a nugget effect to the simulation model degrade the quality of the isotropic by group kernel predictor but it stays close to the quality of the anisotropic. The comparison of the predictive power on the test function shows that kriging with the isotropic kernel is poor. Kriging with an isotropic by group kernel improves the predictive power compared to an anisotropic kernel. To conclude, the proposed methods enable to improve the prediction quality in the context of time-consuming simulation in high dimension.

In this paper, the algorithms are used for an isotropic by group kernel but these methods are generic and could be used for other kernels. Our current work consists in adapting Algorithm 4 to group product kernels, additive kernels presented in Muehlenstaedt et al. (2012) and anova kernels described in Durrande et al. (2013). The clustering method used to group the variables is hierarchical and only focus on the value of the range parameters. An idea is to find a specific method in the case of range parameters and that takes into account the number of inputs in the groups. The estimation of the range parameters needs an optimization of the log likelihood function. At each step of the algorithm, the chosen parameter set for initialization of the optimization is the point that maximizes the log likelihood function among a space filling design. The idea is to initialize the optimization with the value of range parameters estimated at the previous step. In the paper of Welch et al. (1992), they propose a modification in their algorithm to accelerate the optimization. They optimize on only one range parameter. This modification could be apply in our algorithms.

\section{Acknowledgments}

This work benefited from the financial support of the ANR project "PEPITO" (ANR-14-CE230011) 


\section{Bibliography}

Antoniadis, A., Helbert, C., Prieur, C., and Viry, L. (2012). Spatio-temporal metamodeling for West African monsoon. Environmetrics, 23(1):24-36.

Binois, M. (2015). Uncertainty quantification on pareto fronts and high-dimensional strategies in bayesian optimization, with applications in multi-objective automotive design. PhD thesis, Ecole Nationale Supérieure des Mines de Saint-Etienne.

Binois, M., Ginsbourger, D., and Roustant, O. (2015). Quantifying uncertainty on Pareto fronts with Gaussian process conditional simulations. European J. Oper. Res., 243(2):386-394.

Booker, A. J., Dennis, Jr., J. E., Frank, P. D., Serafini, D. B., and Torczon, V. (1998). Optimization using surrogate objectives on a helicopter test example. In Computational methods for optimal design and control (Arlington, VA, 1997), volume 24 of Progr. Systems Control Theory, pages 49-58. Birkhäuser Boston, Boston, MA.

Cornford, D., Nabney, I. T., and Williams, C. K. I. (2002). Modelling frontal discontinuities in wind fields. J. Nonparametr. Stat., 14(1-2):43-58. Statistical models and methods for discontinuous phenomena (Oslo, 1998).

Cressie, N. A. C. (1993). Statistics for spatial data. Wiley Series in Probability and Mathematical Statistics: Applied Probability and Statistics. John Wiley \& Sons, Inc., New York. Revised reprint of the 1991 edition, A Wiley-Interscience Publication.

Durrande, N. (2001). Étude de classes de noyaux adaptées à la simplification et à l'interprétation des modèles d'approximation. Une approche fonctionnelle et probabiliste. $\mathrm{PhD}$ thesis, Ecole Nationale Supérieure des Mines de Saint-Etienne.

Durrande, N., Ginsbourger, D., and Roustant, O. (2012). Additive covariance kernels for highdimensional Gaussian process modeling. Ann. Fac. Sci. Toulouse Math. (6), 21(3):481-499.

Durrande, N., Ginsbourger, D., Roustant, O., and Carraro, L. (2013). ANOVA kernels and RKHS of zero mean functions for model-based sensitivity analysis. J. Multivariate Anal., 115:57-67.

Everitt, B. S., Landau, S., Leese, M., and Stahl, D. (2011). Cluster analysis. Wiley Series in Probability and Statistics. John Wiley \& Sons, Ltd., Chichester, fifth edition.

Fricker, T. E., Oakley, J. E., and Urban, N. M. (2013). Multivariate Gaussian process emulators with nonseparable covariance structures. Technometrics, 55(1):47-56.

Gao, J., Gunn, S., and Kandola, J. (2002). Adapting kernels by variational approach in SVM. In AI 2002: Advances in artificial intelligence, volume 2557 of Lecture Notes in Comput. Sci., pages 395-406. Springer, Berlin.

Ginsbourger, D., Roustant, O., Schuhmacher, D., Durrande, N., and Lenz, N. (2016). On ANOVA decompositions of kernels and Gaussian random field paths. In Monte Carlo and quasi-Monte Carlo methods, volume 163 of Springer Proc. Math. Stat., pages 315-330. Springer, [Cham].

Marrel, A., Iooss, B., Van Dorpe, F., and Volkova, E. (2008). An efficient methodology for modeling complex computer codes with Gaussian processes. Comput. Statist. Data Anal., 52(10):4731-4744. 
Muehlenstaedt, T., Roustant, O., Carraro, L., and Kuhnt, S. (2012). Data-driven Kriging models based on FANOVA-decomposition. Stat. Comput., 22(3):723-738.

Paciorek, C. J. and Schervish, M. J. (2006). Spatial modelling using a new class of nonstationary covariance functions. Environmetrics, 17(5):483-506.

Padonou, E. and Roustant, O. (2016). Polar Gaussian processes and experimental designs in circular domains. SIAM/ASA J. Uncertain. Quantif., 4(1):1014-1033.

Rasmussen, C. E. and Williams, C. K. I. (2006). Gaussian processes for machine learning. Adaptive Computation and Machine Learning. MIT Press, Cambridge, MA.

Roustant, O., Ginsbourger, D., and Deville, Y. (2012). Dicekriging, diceoptim: Two r packages for the analysis of computer experiments by kriging-based metamodeling and optimization.

Santner, T. J., Williams, B. J., and Notz, W. I. (2003). The design and analysis of computer experiments. Springer Series in Statistics. Springer-Verlag, New York.

Schwarz, G. (1978). Estimating the dimension of a model. Ann. Statist., 6(2):461-464.

Snelson, E. L. (2008). Flexible and efficient Gaussian process models for machine learning. ProQuest LLC, Ann Arbor, MI. Thesis (Ph.D.)-University of London, University College London (United Kingdom).

Stein, M. L. (1999). Interpolation of spatial data. Springer Series in Statistics. Springer-Verlag, New York. Some theory for Kriging.

Stitson, M., Gammerman, A., Vapnik, V., Vovk, V., Watkins, C., and Weston, J. (1997). Support vector regression with anova decomposition kernels. Advances in kernel methodsSupport vector learning, pages 285-292.

Sudret, B. (2012). Meta-models for structural reliability and uncertainty quantification. arXiv preprint arXiv:1203.2062.

Villa-Vialaneix, N., Follador, M., Ratto, M., and Leip, A. (2012). A comparison of eight metamodeling techniques for the simulation of $\mathrm{n} 2$ o fluxes and $\mathrm{n}$ leaching from corn crops. Environmental Modelling \& Software, 34:51-66.

Welch, W. J., Buck, R. J., Sacks, J., Wynn, H. P., Mitchell, T. J., and Morris, M. D. (1992). Screening, predicting, and computer experiments. Technometrics, 34(1):15-25.

Yi, G. (2009). Variable selection with penalized Gaussian process regression models. PhD thesis, University of Newcastle Upon Tyne. 

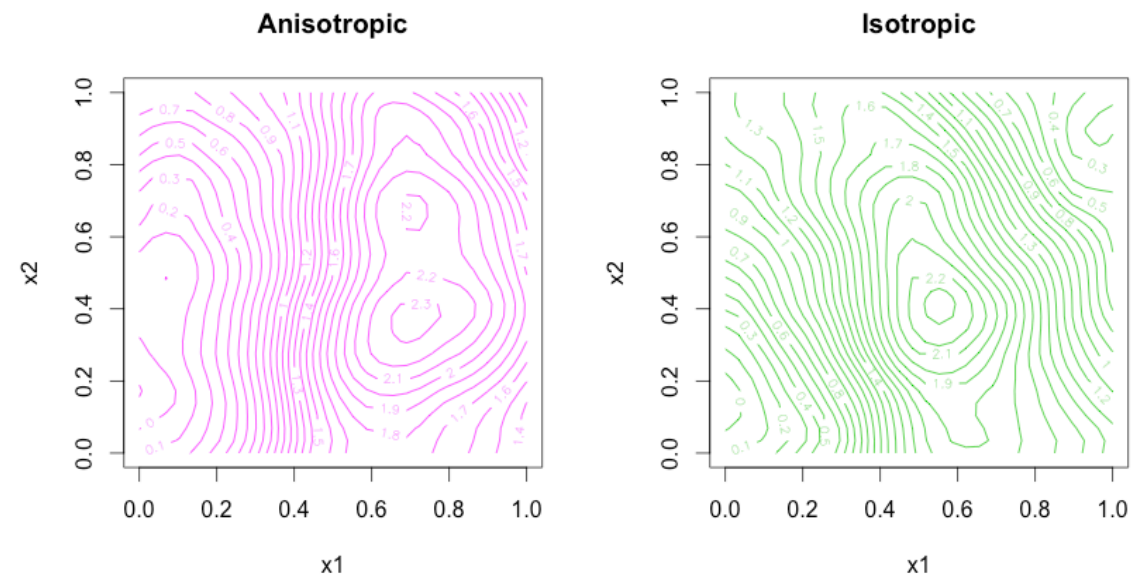

Figure 7: Simulation of a Gaussian process trajectory with 2 different kernels. Anisotropic on the left and isotropic on the right.

\section{Annexes}

\section{A Visualization of isotropic and anisotropic kernels}

We simulate in the Figure 7 a Gaussian process with $p=2$ parameters, the simulated model is

$$
Y(\mathbf{x})=1+\epsilon(\mathbf{x})
$$

where $\epsilon$ is a Gaussian process with a standard deviation $\sigma^{2}=1$.

With an anisotropic kernel:

$$
r_{\boldsymbol{\theta}}\left(\mathbf{x}-\mathbf{x}^{\prime}\right)=\rho_{\theta}\left(\left|x_{1}-x_{1}^{\prime}\right|\right) \times \rho_{\theta}\left(\left|x_{2}-x_{2}^{\prime}\right|\right)
$$

and an isotropic kernel :

$$
r_{\boldsymbol{\theta}}\left(\mathbf{x}-\mathbf{x}^{\prime}\right)=\rho_{\theta}\left(\left\|\mathbf{x}-\mathbf{x}^{\prime}\right\|_{2}\right)
$$

where $\rho_{\theta}$ is a Matern5_2 and $\theta=0.5$.

\section{B Algorithm W}

This algorithm is inspired by Welch et al. (1992). At each step $k$, if the previous model has $s_{k-1}=k-1$ groups, the next estimated models will have $s_{k}=k$ groups.

We note $\mathcal{M}_{s_{k}}$ the model at step $k$ with $s_{k}=k$ groups. Let $\mathcal{I}_{1}=\left\{i_{1,1}, \ldots, i_{1, p_{1}}\right\} ; \mathcal{I}_{2}=$ $\left\{i_{2,1}\right\} ; \ldots ; \mathcal{I}_{s_{k}}=\left\{i_{s_{k}, 1}\right\}$, with $p_{1}+s_{k}-1=p$ where $\left(s_{k}-1=k\right)$.

\section{Checking step:}

We create an additional group in taking an index $i_{1, m} \in \mathcal{I}_{1}, m=1, \ldots, p_{1}$ out of the group 1 . We have $J_{k}=p_{1}$ possibilities and we obtain $s_{k}=s_{k-1}+1$ groups. we create $J_{k}$ models noted $\mathcal{M}_{s_{k}}^{1}, \ldots, \mathcal{M}_{s_{k}}^{J_{k}}$ and we follow the General step.

In the Algorithm 2 steps 5-9 are replace by : 


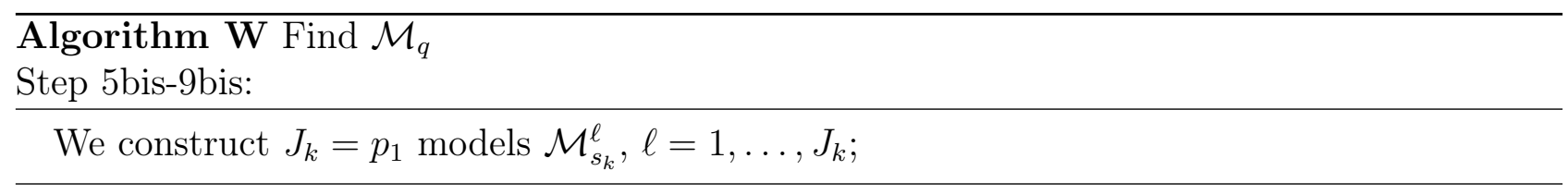

\section{Code}

The code of the algorithms is developed on $R$. It includes some $C$ code completely integrate in the $R$ code. The entire code and the examples used in the application section is available on the github or in the electronic appendix.

The package kergp is used to construct the $\mathrm{C}$ class of the isotropic by group kernel. All the functions needed to compute the four algorithms is on the same file named fonctions.R. The four algorithms are in four files algo1Base.R, algo2Base.R, algo3Base.R, algo4Base.R and algoWelch.R. The functions are describes in the table 7 . The algorithms code are parallelized when it is possible. The hierarchical clustering method is done by the function hclust available in the package stats. The function used to cut the dendrogram to obtain the best composition of groups best.cutree is available in the package JLutils. Finally, the code to run the simulations of the applications is on the files given by the table 7. The file DataVisu.R is used to condition the data before visualization.

\begin{tabular}{l|ccc} 
names & class & description & default value \\
\hline $\mathrm{x}$ & data.frame & Design & NA \\
$\mathrm{y}$ & vector & Response & NA \\
crit & function & AIC, BIC or tune & NA \\
stockage & boolean & Data storage or not & FALSE \\
itmax & integer & maximum iteration number & 500 \\
ncores & integer & number of cores used & 1
\end{tabular}

\begin{tabular}{l|cc} 
names & class & description \\
\hline best & list & groups composition \\
critere & matrix (size $1 \times 1)$ & Criterion value (BIC, AIC or tune) \\
model & gp & GP model \\
time & proc_time & Estimation time \\
Stock & list & storage of model estimate at each step \\
& character & "No storage"
\end{tabular}

Table 6: Description of the functions :algo1Base.R, algo2Base.R, algo3Base.R, algo4Base. $R$ and algoWelch.R

\begin{tabular}{l|ccc} 
Applications & section & file R & parallelized \\
\hline Comparison & 4.1 .1 & Bench.R & Yes \\
Learning set size & 4.1 .2 & Size.R & Yes \\
Error added on parameters & 4.1 .3 & Err.R & Yes \\
nugget effect & 4.1 .4 & Nugg.R & Yes \\
Sobol function & 4.2 & Sobol.R & No
\end{tabular}

Table 7: Files to run the applications

The four first examples need the file DataVisu.R to clean the data for the visualization. 